Available online on 15.01.2020 at http://jddtonline.info
Open Access to Pharmaceutical and Medical Research
unrestricted non-commercial use, provided the original work is properly cited

Open@ Access

Review Article

\title{
Cubosomes: A Novel Carrier for Transdermal Drug Delivery
}

\author{
Dhadwal Avantika *, Sharma Dev Raj, Pandit Vinay, Ashawat Mahendra Singh, Kumar Pravin \# \\ Department of Pharmaceutics, Laureate Institute of Pharmacy, Kathog, H.P-176031
}

\begin{abstract}
Cubosomes are square and rounded particles with internal cubic lattice. Cubosomes are thermodynamically stable and consist of honeycombed (cavernous) structures separating two internal aqueous channels and a large interfacial area. Cubosomes are nanoparticles which are self assembled liquid crystalline particles of certain surfactants with proper ratio of water with microstructure that provides unique properties of practical interest. Bicontinuous cubic liquid crystalline phase is optically clear and very viscous material has the unique structure at nanometer scale. The word bicontinuous refers to the division of the two continuous but non-intersecting aqueous regions by lipid bilayer that is twisted into space filling structure. Hydrating a surfactant or polar lipid that forms cubic phase and then dispersing a solid like phase into smaller particles usually forms a cubosomes. Self-assembled cubosomes as active drug delivery systems are receiving more and more attention and interest after the first discovery and nomination. They exhibit different internal cubic structure and composition with different drug-loading modalities. It has high internal surface area and cubic crystalline structures, relatively simple preparation method, biodegr adability of lipids, the ability of encapsulating hydrophobic, hydrophilic and amphiphilic substances, targeting and controlled release of bioactive agents. Cubosomes are having wide range of applications in various fields and they can be characterized by various evaluation parameters. So, Cubosomes are gaining more attention in pharmaceutical field.
\end{abstract}

Keywords: Cubosomes, Liquid crystal, drug-loading, hydrophilic, hydrophobic, amphiphilic.

Article Info: Received 30 Oct 2019; Review Completed 19 Dec 2019; $\quad$ Accepted 24 Dec 2019; Available online 15 Jan 2020

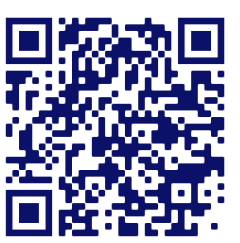

Cite this article as:

Dhadwal A, Sharma DR, Pandit V, Ashawat MS, Kumar P, Cubosomes: A Novel Carrier for Transdermal Drug Delivery, Journal of Drug Delivery and Therapeutics. 2020; 10(1):123-1130 http://dx.doi.org/10.22270/jddt.v10i1.3814

*Address for Correspondence:

Avantika Dhadwal, Department of Pharmaceutics, Laureate Institute of Pharmacy Kathog, H.P-176031 Pravin Kumar- Associate Professor, Head of Department of Pharmaceutics, Laureate Institute of Pharmacy Kathog, H.P-176031

\section{Introduction}

A drug delivery system is a device that safely brings a therapeutic agent to the specific body site at a certain rate to achieve an effective concentration at the site of drug action. The release of drug in a predesigned manner is termed controlled drug release (CR), which is used to promote therapeutic benefits while minimizing toxic side-effects. Sustained release over an extended period of time may reduce the need for multiple dosing which will be a benefit in terms of reduced cost and increased patient compliance [13]. To achieve targeted drug delivery by encapsulating the drug within vesicular structure such a system is known to be vesicular drug delivery system. In this system if the vesicles behave as carrier system it will transport high molecular weight of drugs, and if behave as penetration enhancer it will increase the drug transport rate across the skin $[4,5]$. There are huge number of vesicular drug delivery systems that allow drug targeting and the sustained or controlled release of conventional medicines. In such a system cubosomes are also part of the vesicular drug delivery system or lipid based colloidal system which were discovered in 1980 [4, 5]. The term "Cubosomes" were coined by Larsson that reflects the cubic molecular crystallography and similarity to liposomes $[6,7]$.

Cubosomes are distinct, sub-micron, nano-structured particles of bicontinuous cubic liquid crystalline phase [7, 8]. Cubosomes are nanoparticles which are self assembled liquid crystalline particles of certain surfactants with proper ratio of water with microstructure and also posses as solid like rheology [9]. Cubosomes possess the same microstructure as the parent cubic phase but have larger specific surface area and their dispersions have much lower viscosity in comparison to the bulk cubic phase $[7,10]$. Bulk cubic phases have higher viscosity than cubosomal dispersion [11-13]. Most concentrated surfactants that form cubic liquid crystals lose these phases to micelle formation at high dilutions, due to optimal water insolubility. Their cubic phases exist in equilibrium with excess water and can be dispersed to form cubosomes. Cubosomes are typically produced by high-energy dispersion of bulk cubic phase, followed by colloidal stabilization using polymeric surfactants. One application of cubic phase liquid crystals is the controlled release of selected water-oil soluble molecules. The emulsification of cubic lipid phases in water results in the production of cubosomes that can be defined as 
nanoparticle disperse systems characterized by high biocompatibility and bioadhesivity [14-16]. Cubosomes are composed of lipids, surfactants and polymer molecules which have both polar and non polar components, termed as amphiphilic. The hydrophobic effect drives amphiphilic molecules in polar solvents to spontaneously self assembling into an array of thermodynamically stable liquid crystalline phases with lengths on nanometer scale [17]. Thus, cubosomes are bicontinuous cubic liquid phase enclosing two separate regions of water divided by surfactant controlled bilayers [7]. Bicontinuous cubic phases are optically isotropic, very viscous and solid like liquid crystalline substance having cubic crystallographic symmetry [17]. Cubosomes have great importance in nanodrug formulations [7].

\section{Structure of Cubosomes}

The basic structure of cubosomes includes honeycombed structures separating the two internal aqueous channels along with large interfacial area [Fig 1] [12]. Amphiphilic molecules form bicontinuous water and oil channels, where "bicontinuous" refers to two distinct (continuous, but nonintersecting) hydrophilic regions separated by the bilayer [12]. The structure generally maintains the efficacy; stability of actives such as vitamins and proteins. In the structure, surfactants assembles into bilayers that are twisted into a three dimension, periodic, minimal surface forming tightly packed structure, like "honeycombed" with bicontinuous domains of water and lipid. Its structure is different from liposomes because its can accommodate water-soluble, lipid soluble and amphiphilic molecules [18]. The interconnectiveness of the structure results in a clear viscous gel that is similar in appearance and rheology to cross-linked polymer hydrogels. Monoglyceride-based cubic gels possess long range order than hydrogels and their composition (i.e., lipid and water) gives excellent biocompatibility [12].

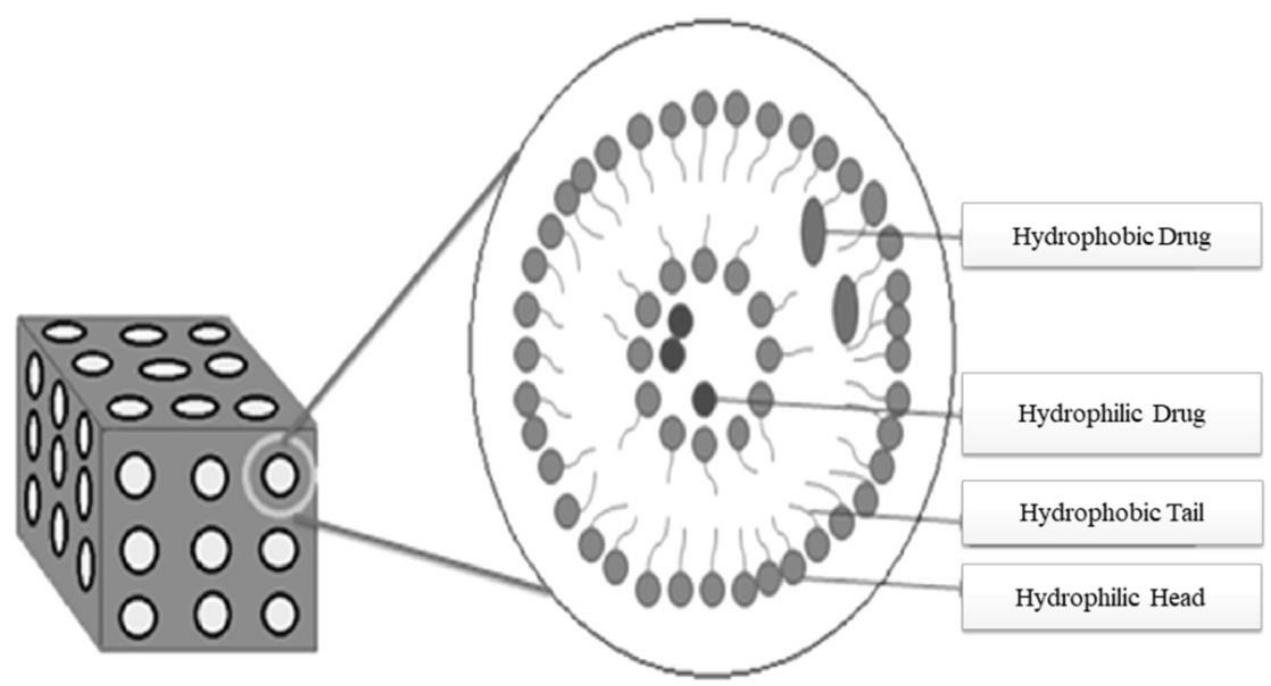

Figure 1: Structure of Cubosome.

\subsection{Types of Cubosomes}

2.1.1. Liquid Cubosome Precursors: The hydrotrope dilution process is found to produce more stable and smaller cubosomes. Nucleation process allows the formation of particles whose growth is seen under crystallization and precipitation processes. Monoolein is properly dissolved in a hydrotrope, such as ethanol, that prevents it from liquid crystalline formation. Thus, dilution of this mixture spontaneously "crystallizes" or precipitates the cubosomes. Quid precursor process is allowed for easier scale up of cubosome preparations and avoids the bulk solids handling and potentially damaging high energy processes $[19,20]$.

\subsubsection{Powdered Cubosome Precursor:}

Powdered cubosome precursors are composed of dehydrated surfactant coated with polymer. Such powders offer advantages to liquid phase hydrotropic cubosome precursors. Cubosomes with a mean particle size of $600 \mathrm{~nm}$ are formed by the hydration of the precursor powders, as confirmed by light scattering and cryo-TEM [10]. Cubosomes which are made up with the use of lipids are waxy and sticky solids. Coating of the waxy lipid on water-soluble noncohesive starch generally prevents from agglomeration and controls the size of the particle. An excellent process for his purpose is spray drying. [20].

\section{Structural components of cubosomes}

\subsection{Amphiphilic lipids}

The widely used amphiphilic lipids to prepare cubosomes are gyceryl monooleate GMO, usually discussed to as monoolein and phytantriol (PHYT) [21, 22]. GMO consists of mixture of the glycerides of oleic acid and other fatty acids, containing mostly of monooleate which fits to the group of amphiphilic lipids with capacity to form various lyotropic liquid crystals [23, 24]. It has been reported that GMO with hydrocarbon chain length in the range of 12-22 have greater tendency to form cubic phases [25]. Further, GMO is a biocompatible and biodegradable material under as generally recognized as safe (GRAS) category by FDA, mainly used as emulsifier in the food industry. PHYT is a material with a phytanyl chain, also exhibit the formation of cubic phases upon increasing the water content. PHYT, chemically 3,7,11,15-tetramethyl-1,2,3-hexadecanetriol, is a frequently used component in cosmetic products. It has been recommended as a good alternate for GMO in the manufacturing of cubosomes. As compared to GMO, PHYT has an added advantage that it shows greater structural 
stability. The GMO are more prone to ester hydrolysis [26]. Although PHYT and GMO have different molecular structures, these two materials show much related phase transition behaviors with increasing water content and temperature.

\subsection{Stabilizers}

The research scientists have suggested an important role for the surfactants as stabilizers for improved stability of cubosomes against coalescence to the bulk cubic phase. Poloxamer 407 (P407), a PEO-PPO-PEO tri-block copolymer, is mostly investigated surfactant in the preparation of cubosomes with its PPO portions being located either at the surface of the cubosomes or within the bilayer structure, whereas the PEO chains are exposed to the surrounding water phase $[27,28]$. P407 is usually applied upto a concentration of $20 \% \mathrm{w} / \mathrm{w}$ depending on the quantity of dispersed phase. Wadsten-Hindrichsen et al. [29] investigated the effect of three water-miscible solvents i.e propylene glycol (PG), polyethylene glycol 400 (PEG400) and 2-meth-yl-2,4-pentanediol (MPD) on a PHYT-based cubosomal system. They showed that MPD produced a sponge phase whereas with the two other solvents, PG and PEG400, only cubic, lamellar and non-ordered liquid phases were identified. The main reasons attributed for difference in phase behavior were more hydrophobic nature of PHYT as compared to GMO and the branched hydrocarbon chain of PHYT, which makes PHYT less flexible than GMO.

On substituting b-casein with P407 as the stabilizer to GMO and PHYT based cubosomes, its internal structure as well as its morphology was studied [30]. A Pn3 m phase structure was displaced by the GMO-b-casein cubosomes and at $60^{\circ} \mathrm{C}$ a QII to HII phase transition .Whereas, Im3 $\mathrm{m}$ phase structure has P407-GMO dispersion and at higher temperatures, that is, $70^{\circ} \mathrm{C}$ the HII phase gets appeared. The concentration of the stabilizer and the $\mathrm{Pn} 3 \mathrm{~m}$ phase structure was detected in the case of PHYT systems. P407-PHYT dispersion displayed only a direct QII to La conversion, whereas upon heating the b-casein-PHYT system displayed a QII to HII to La transition behavior. Steric stabilization to dispersions of lipid nanostructured particles gives the bcasein and Im3m structure in nanoparticles avoids the transition. In cubosomes, it was discovered that the poly (ethylene oxide) stearate stabilizers (commercially known as Myrj) were more effective as steric stabilizers. In contrast, Myrj 59 was proven to be more effective than P407 for PHYT cubosomes with an average of 100 poly (ethylene oxide) units at a concentration of fivefold lower than that of P407 [46]. To GMO-based cubosomes with P407 the hydroxypropyl methylcellulose acetate succinate (HPMCAS) showed roughly equal stability [31].

\section{Advantages and limitations of cubosomes:}

\subsection{Advantages of cubosomes}

1. They have ability to encapsulate both hydrophilic and hydrophobic also amphiphilic drugs.

2. They have a sustained- release drug delivery characteristics.

3. Cubosomes have biocompatibility and bioadhesivity properties.

4. Bicontinuous cubic liquid crystalline phase of cubosomes even stable in excess water [32].

5. Cubic phase materials can be formed by simple combination of biologically compatible lipids and water and are thus well suited for use in treatments of skin, hair, and other body tissue.
6. With respect to liposomes, cubosomes possesses a larger ratio between the bilayer area and the particle volume and a larger breaking resistance [33]

7. Because of their high internal surface area and crystalline cubic structures they have high drug payloads.

8. They can be prepared by simple method and possess lipid biodegradability [34, 35].

9. Targeted release and controlled release of bioactive agents [7].

10. Cubosomes are excellent solubilizers, compared with conventional lipid or non-lipid carriers.

11. They show high drug carrier capacity for a range of sparingly water-soluble drugs.

12. These are an excellent vehicle to protect the sensitive drug from enzymatic degradation and in-vivo degradation, such as peptides and proteins.

13. The cuboidal system enhances the bioavailability range twenty to more than one hundred times of water-soluble peptides [36].

14. The cubic phases of cubosomes can be fractured and dispersed to form particulate dispersions that are colloidally and/or thermodynamically stable for longer time [7].

\subsection{Limitation of cubosomes as a drug carrier}

1. Cubosomes may lead to low drug loading efficiency and drug leakage in preparation, preservation and transport in vivo, thus the major problem of their stability acts as a barrier and thus limiting their use [37].

2. Large scale production is sometimes difficult because of high viscosity [7].

\section{Manufacturing of Cubosomes}

Cubosomes can be manufactured by two distinct methods:

5.1 Top down approach: In the research area they are the most widely used methods. It was reported in 1996 by Ljusberg-Wahren [Fig 2]. The viscous bulk cubic phase is prepared by mixing the lipids with stabilizers; by the input of high energy (such as High-Pressure Homogenization $[\mathrm{HPH}]$, sonication or shearing) the resultant mixture is dispersed into aqueous solution to form Lyotropic Liquid Crystal (LLC) nanoparticles. A clear rigid gel formed by water-swollen cross-linked polymer chains that resembles the bulk cubic phase. In the preparation of LLC nanoparticles, HPH is the most widely used technique. In top-down approach, cubosomes are prepared and observed to co-exist with vesicles (dispersed nanoparticles of lamellar liquid crystalline phase) or vesicle-like structures [35].

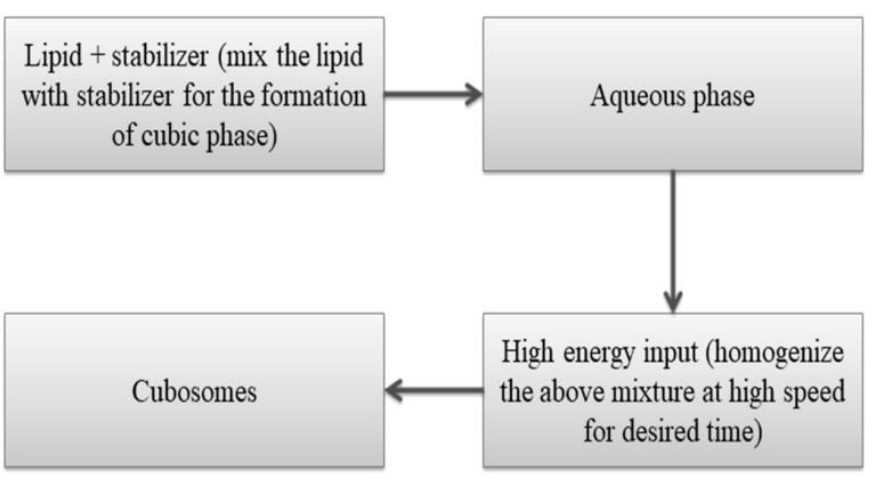

Figure 2: Cubosomes by Top down Technique. 
5.2 Bottom up approach: In the bottom-up approach it forms the nanostructure building blocks and then assembles them into the final material [Fig 3]. In water insoluble lipids the hydrotrope gets dissolves so as to create a liquid precursors. Less energy input is needed at high concentration to prevent the formation of liquid crystals. Dispersing inverse micellar phase droplets leads to the formation of cubosomes in water at $80^{\circ} \mathrm{C}$ and allow them to slowly cool and droplets get crystallizes to cubosomes. Monoolein-ethanol solution is diluted with aqueous poloxamer 407 solution produced by cubosomes at room temperature. Thus cubosomes are formed by emulsification. The bottom-up approach cannot avoid the forming of vesicles through cryo-TEM, many vesicles and vesicle-like structures were also observed to coexist with cubosomes [20].

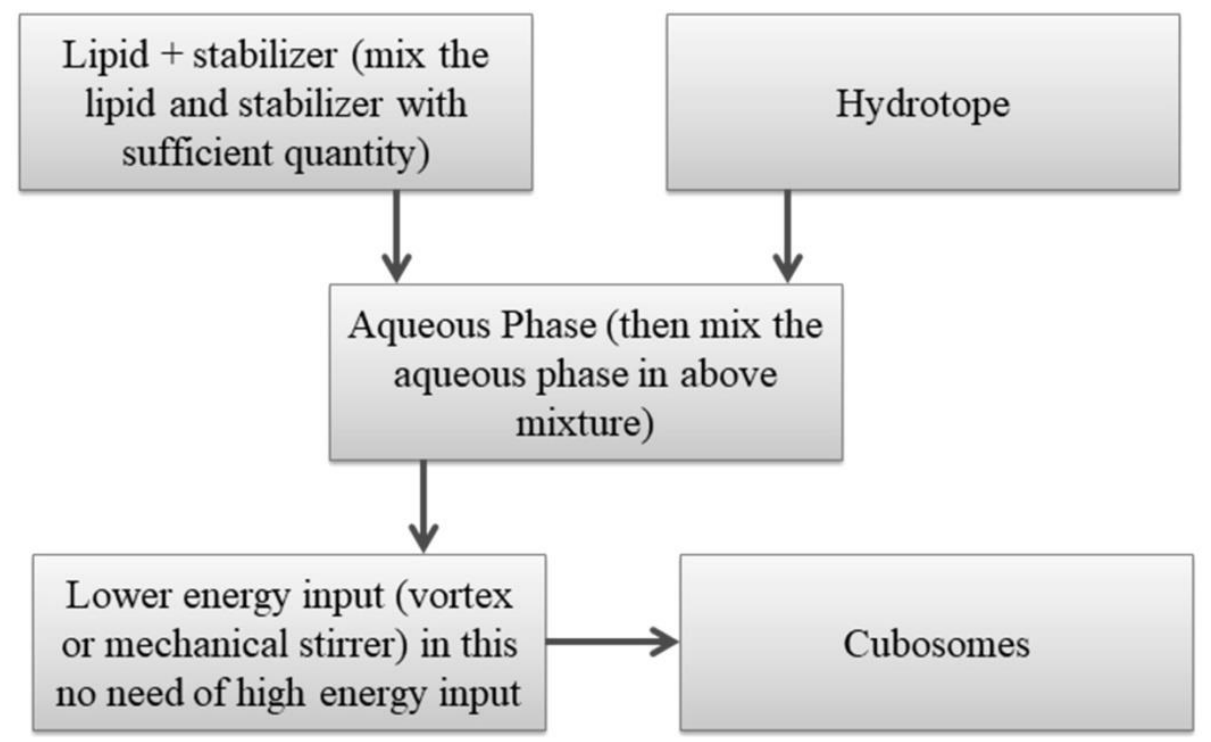

Figure 3: Cubosomes by Bottom up Technique.

\section{Characterization of drug release from cubosomes}

For maintaining the concentrations of the drug in the therapeutic concentration range for a prolonged period of time, the sustained release drug delivery systems are beneficial. It helps in the reductions of frequency of drug administration and avoids hazardous side effects. Based on the two main rationales ,the cubosomes have been proposed as a delivery system for drugs and other bioactive agents:

- $\quad$ solubilization of poorly water-soluble drugs

- controlled or sustained release of the loaded drugs

The release of drugs of various molecular weights and polarities are controlled and incorporated by the cubic phase. Higuchi-type diffusion-controlled kinetics is obeyed by the cubic phase matrix from where the drug releases and shows a linear dependence upon the square root of time where diffusion is out of the matrix.

During the diffusion of incorporated drugs, their surface area generally enlarges because of the bulk cubic phase that gets divided into dispersed nanostructured particles as cubosomes. On division the main properties of cubic phase (i.e. solubility, drug loading and diffusion of drug through the cubic matrix) are not expected to change into cubosomes. The cubosomes with a surface area of a diameter of about $200 \mathrm{~nm}$ which is actually 200000 times much more greater than the equivalent cubic part of cubic phase, have greater diffusion rate of the drug. In controlled-release system, it is difficult to apply it directly because of the nanoscale size of the cubosomes [38]. To prevent rapid drug release their charge, viscosity and structure seems to be very useful. The physicochemical properties of the drug, swelling capacity, interaction between the nanocarrier and active agent, structure and composition of the lipid bilayers, all depends on their release profile. From cubosomes, Lai et al. [39] studied the release of simvastatin, which is a hydrophobic active. In gastric and intestinal fluids the simvastatin crystal simulated a rapid release of more than $90 \%$ at $1 \mathrm{hr}$ and in the same medium at $10 \mathrm{hr}$ the cubosomes were released to less than $3 \%$ of simvastatin. In the cubic matrix the hydrophobic portion shows the high affinity of simvastatin that prevents the drug from escaping into the nanostructured particles. The two different cubosomes, prepared from GMO and PHYT, where studied by Lee et al. [40]. Various molecular weights were evaluated for several model hydrophilic drugs for in-vitro release kinetics and matrix was thus changed from QII GMO to QII PHYT that release the rates of drug which got decreased. The sustained release of cinnarizine over $48 \mathrm{~h}$ after oral administration to rats were demonstrated by cubosomes formed from PHYT was reported by Nguyen et al. [41] . When the cubosomes were prepared by using GMO, its drug plasma concentrations fell below after $24 \mathrm{hr}$. The incorporation of some small and large molecules whose enlarged size on release profile from the cubic phase of GMO was investigated by the Clogston et al. [42]. Thus, when the size of the added compound increases the rate of release decreases. Rubipy and tryptophan, are considered as small molecules whose release from the cubic phase over a period of time is 1-3 days, whereas cytochrome c, lysozyme and myoglobin, which are mid-sized molecules, they generally occur in a week. Ovalbumin, conalbumin, apo-ferritin and calf thymus DNA, are the large macromolecules which occur in 3 weeks with less than $12 \%$ of the compound. 


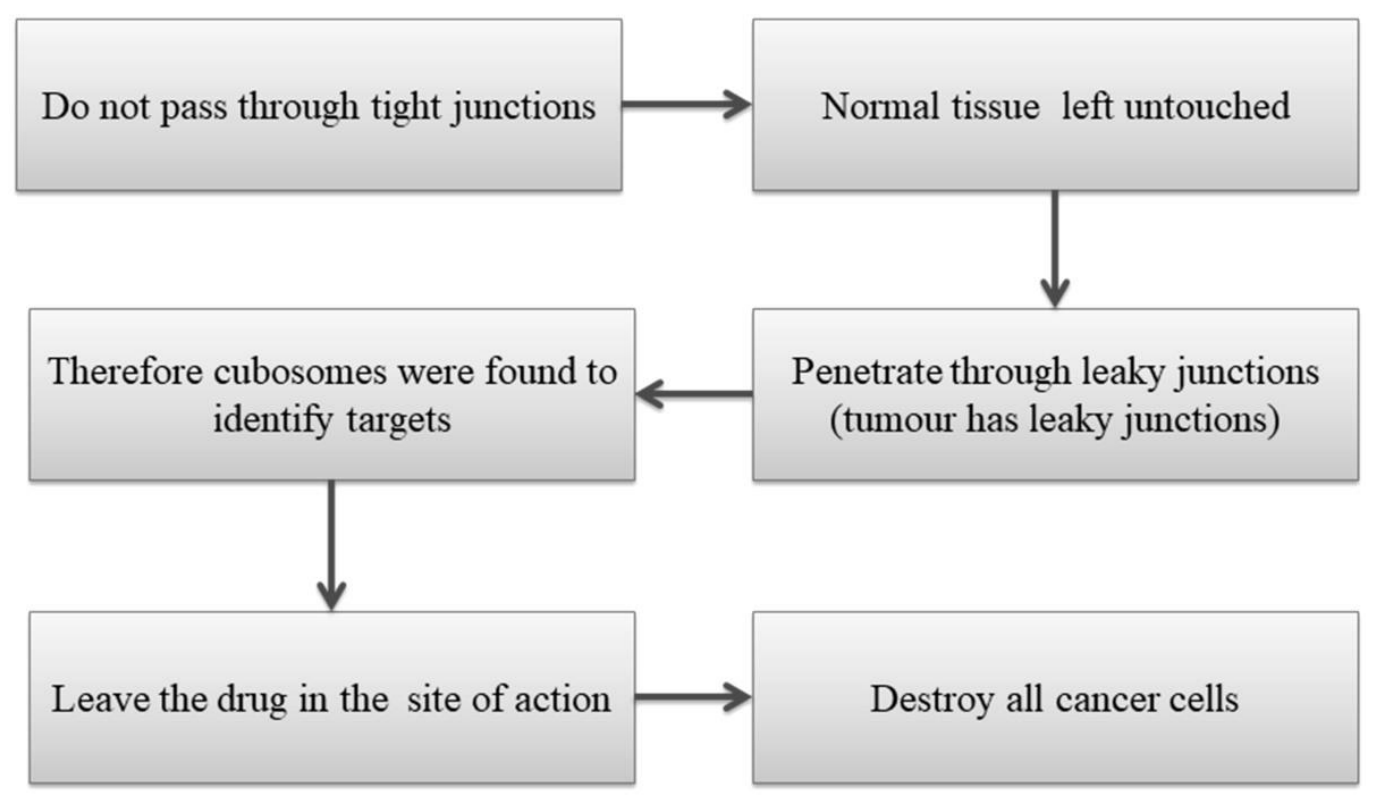

Figure 4: Action of Cubosomes incorporated drugs on tumors.

\section{Opportunity for improvement of loading and release behavior of cubosomes through functionalization}

The loading of drugs in nanocarriers and/or the release behavior of loaded drugs are controlled by modifying the cubosomes with concept of functionalization. As most of the carrier is occupied by water, the loading of water soluble molecules becomes challenging due to relatively fast drug leakage. Surfactants serving as anchors there, functionalization of cubosomes can be achieved by integrating into the liquid crystal-the hydrophilic channels are attracted to the hydrophilic part while hydrophobic segment further penetrates into the bilayers of the structure. Surfactant interaction with the hydrophilic drugs can be controlled by adjusting the hydrophilic segments. For example, the loading of an oppositely charged active can be enhanced by a charged part thus the loaded active leads to slower release. Into the LLC, the loading of water-soluble ketoprofen with negative charge was enhanced by using surfactants of positive charge such as dimethyl ammonium chloride, dioctadecyl dimethyl ammonium chloride and dioctadecyl ammonium chloride [43]. The amount of surfactant added for functionalization is critical one because, larger amount can change the cubic phase into lamellar phase. Gurfinkel et al reported that if the concentration of oleyl lactate (OL) is increased, it will affect the lipid packing as well as hydrate the head groups. At low concentration the cubic phase was formed, whereas at higher concentration the hexagonal phase was preferred [43].

The matrix of cubic phase is affected by the location of the surfactants. Although within the lipid bilayer the more lipophilic types prefers to stay, thus leading to the formation of lamellar phase, also at its interfacial area the more polar types locates which directs the formation of reverse hexagonal phase [44]. In this way, a functionalizing surfactant molecule must have reachable hydrophilic part, low water solubility and Krafft temperature, and critical packing parameter (head-to-tail volume ratio) equal to unity Liu et al. [45] showed that the phase transition from cubic phase to lamellar phase of PHYT occurs when the curvature of the self-assembled system toward the hydrophobic section increased and the ionic surfactant content increases. Similarly, when the phase transition from lamellar phase to cubic phase occurs, it occurs between the head groups of the ionic surfactant where the ionic strength increased leading to the decrease in the repulsion. Because of various hydrophilic-lipophilic balance (HLB) values the cubic-phasebased drug delivery system is crucial for the selection of additives. By varying the polarity of the additives the phase behavior of GMO was studied [46]. To improve the efficiency of GMO-based drug delivery systems the additives were added with low HLB that promoted higher and faster transformation into the cubic phase upon hydration. Thus, for bioadhesive formulations, the lamellar phase with additives of high HLB induces the formation of GMO. When large amphiphilic polymers or 'tethers' are employed into the matrix of cubosomes they leads to the approaches of the functionalization. Not just on the surface of the cubosomes and on the internal bilayers of the cubic matrix, Landh [47] used large amounts of P407 to incorporate into it. Most of the polymers adhere to the surface of the particles at low concentrations of P407 as proven; whereas the phase transition occur from Pn $3 \mathrm{~m}$ to $\mathrm{Im} 3 \mathrm{~m}$ at higher concentration and without destroying it the P407 get inserted into the bulk of the cubic matrix [48]. By using a layer-by-layer approach, the seven polyelectrolyte were prepared with polymercoated cubosomes and the burst-release was effectively able to reduce associated with fluorescein-loaded cubosome [49]. Also, pH-sensitive GMO based cubic phase was developed when complex coacervation were taken between hydrophobically modified alginate and hydrophobically modified silk fibroin in the water channels [50].The water channels of the cubic phase were blocked by coacervate under low $\mathrm{pH}$ and thus release is prevented. With a higher release, the dissolution of the coacervate results from the higher medium. The initial release rate of a hydrophilic drug from GMO-based cubosomes is retarded through adsorption of a layer of cationic poly-e-lysine by simple surface modification [51]. By incorporating lipopolymers, the internal nanostructures of cubosomes in hydrophilic domains can be functionalized. of can be functionalized. In the water-PHYT inter-facial area the lipopolymers can be incorporated by the PEGylation of the cubosomes using DSPE-mPEGs, thus the internal structures of the nanoparticles gives major effect when induced [52].The internal cubic phases of hydrophilic nanochannels are enlarged due to the presence of DSPE-mPEGs. Therefore, the 
modification of cubic dispersions is an attractive approach for these PEGylated lipids.

\section{Application of Cubosomes}

8.1. Controlled release of drugs: Control release of solubilised substance is the most popular application of cubosomes. Cubic phase is more applicable for control release because of its small pore size $(5-10 \mathrm{~nm})$, ability to solubilize hydrophilic, hydrophobic, amphiphilic molecules and its biodegradability by simple enzymes [Table 1] [53].

Table 1: List of drugs incorporated in Cubosome for Sustained drug delivery system [12]

\begin{tabular}{|c|c|c|c|c|}
\hline S. No. & Researcher & Drug & Category & Associated Disease \\
\hline \multirow[t]{3}{*}{1.} & \multirow[t]{3}{*}{ Engstrom et al, } & $\begin{array}{l}\text { 2-amino-1- } \\
\text { phenylpropanol } \mathrm{HCl}\end{array}$ & Antidepressant & Mania, Depression \\
\hline & & Nitroglycerin & Anti-anginal & Angina pectoris \\
\hline & & Oestriol & Hormonal therapy & $\begin{array}{l}\text { Atrophic vaginitis, } \\
\text { Pruritus }\end{array}$ \\
\hline \multirow[t]{3}{*}{2.} & \multirow[t]{3}{*}{ Sadhale et al. } & Cefazolin & Antibiotics & $\begin{array}{l}\text { Genito-urinary, } \\
\text { respiratory track } \\
\text { infection }\end{array}$ \\
\hline & & Cefuroxime & Antibiotics & $\begin{array}{l}\text { Meningitis bone and soft } \\
\text { tissue infection }\end{array}$ \\
\hline & & Prilocaine & Local anesthetic & In Destistry \\
\hline 3. & Damani & $\begin{array}{l}\text { Clindamycin } \\
\text { phosphate }\end{array}$ & Antibiotics & $\begin{array}{l}\text { Pertonitis, Staphylococcal } \\
\text { bone and joint infection }\end{array}$ \\
\hline 4. & Engstrom et al. & Clomethiazole & Psychotropic & Insomnia \\
\hline 5. & Engstrom et al. & Clotrimazole & Antifungal & $\begin{array}{l}\text { Vagina, mouth and } \\
\text { skin infection }\end{array}$ \\
\hline \multirow[t]{2}{*}{6.} & \multirow[t]{2}{*}{ Engstrom et al. } & Gramicidin & Topical steriod & $\begin{array}{l}\text { Corticosteroid sensitive } \\
\text { dermatoses }\end{array}$ \\
\hline & & Insulin & $\begin{array}{l}\text { Hypo/Hyper } \\
\text { glycaemics }\end{array}$ & Diabetes mellitus \\
\hline \multirow[t]{3}{*}{7.} & \multirow[t]{3}{*}{ Neilsen et al. } & Indomethacin & NSAIDS & Gout, rheumatoid arthritis \\
\hline & & Isosorbidemononitrate & Anti-anginal & Angina pectoris \\
\hline & & $\begin{array}{l}\text { Lidocaine } \\
\text { hydrochloride }\end{array}$ & Oral prepration & $\begin{array}{l}\text { Fungal infection } \\
\text { of external ear }\end{array}$ \\
\hline \multirow[t]{4}{*}{8.} & \multirow[t]{4}{*}{ Boyd } & Diazepam & Sedative-hypnotic & $\begin{array}{l}\text { Anxiety, insomnia, } \\
\text { seizures }\end{array}$ \\
\hline & & Rifampicin & $\begin{array}{l}\text { Bactericidal } \\
\text { antibiotic }\end{array}$ & Tuberculosis \\
\hline & & Griseofulvin & Antifungal & $\begin{array}{l}\text { Fungal infection } \\
\text { of skin }\end{array}$ \\
\hline & & Propofol & Hypnotic & $\begin{array}{l}\quad \text { Procedural sedation, to } \\
\text { induce and maintain } \\
\text { General Anesthesia }\end{array}$ \\
\hline
\end{tabular}

8.2. Melanoma (cancer) therapy: Recently few anticancer drugs have been successfully encapsulated in cubosomes and characterized physicochemically [Fig 4]. The unique structure of this promising nanocarrier suggests its application in melanoma therapy [54].

8.3. Oral drug delivery: Cubosomes address the varied challenges in oral delivery of numerous promising ISSN: 2250-1177 compounds including poor aqueous solubility, poor absorption and large molecular size. These are both liquid and powder in capsule product comprising our self emulsifying liquid crystalline nanoparticles technology (LCNP). In an alternative application large proteins have been encapsulated for local activity in gastrointestinal track. Liquid crystalline nanoparticles technology carriers can be 
combined with controlled release and targeting functionalities. The particles are designed to form in situ in a controlled rate, which enables an effective in vivo distribution of the drug. Liquid crystalline nanoparticles technology carriers can also be released at different absorption sites, for example in upper or lower intestine, which is important for the drugs that have narrow regional absorption window [55]

8.4. Intravenous drug delivery systems: Lipid nanoparticles comprising interior liquid crystal structures of curved lipid membranes are used to solubilize encapsulate and deliver medications to disease areas within the body. While emulsions and liposomes have found use as intravenous carriers in drug products, liquid crystal nanoparticle structures increased payloads of peptides, proteins and many insoluble small molecules and are ideal carriers for injection or infusion of many actives [1].

8.5. Topical drug delivery systems: Cubic phases are more bioadhesive in nature, so that they can conveniently use in topical and mucosal depositions and delivery of different drugs. Topical delivery systems are based on the exploitation of unique properties of liquid crystal (LC) and liquid crystal nanoparticle technologies (LCNT). Topical drug delivery systems are unique in situ forming bioadhesive LC systems facilitate controlled and effective drug delivery to mucosal surfaces (buccal, ophthalmic, vaginal and others). This fascinating system forms a thin surface film at mucosal surfaces consisting of a liquid crystal matrix which nanostructure can be controlled for achieving an optimal delivery profile and provides good temporary protection of sore and sensitive skin $[56,57]$.

\section{Conclusion}

Bicontinuous cubic liquid crystalline phases, either in bulk or cubosome form, offer unique properties of particular interest to the personal care industry. Cubic phase materials can be formed by simple combination of biologically compatible lipids and water and are well used in the treatments of skin, hair, and other body tissue. The main application of cubosomes are in melanoma (cancer) therapy, oral drug delivery systems, intravenous drug delivery systems and topical drug delivery systems. The ability to form cubosomes during formulation, or during manufacture offers great flexibility in the product development. Cubosomes which are prepared in dispersion possess a nanometer scale structure identical to the bulk cubic phase. The bulk cubic phase has sufficient length scale to allow controlled release of solutes. Formulation of personal care products containing cubosomes often requires additional surface-active ingredients. Some observations of skin irritation by bulk cubic phase generally used to avoid residual oleic acid. It is expected that in the next 10 to 20 years, cubosomes containing protein and peptide based drugs will constitute more than half of the new drugs introduced into the market and more than $80 \%$ of these protein drugs will be antibodies due to control release activity. Further specialized studies are required to confirm this fascinating hypothesis and to better investigate the role of vesicles and cubosomes in controlling the release of the drug.

\section{Conflict of Interest:}

The authors declare no conflict of interest.

Acknowledgments: The Authors are thankful to Dr. Ran Singh Chaudhary, Chairman, Laureate Institute of Pharmacy, for providing research facility.

\section{References}

[1] Rarokar NR, Khedekar PB. Cubosomes: a vehicle for delivery of various therapeutic agents. MOJ Toxicol. 2018; 4:19-21.

[2] Shah JC, Sadhale Y, Chilukuri DM. Cubic phase gels as drug delivery systems. Advanced drug delivery reviews. 2001;47:229-250.

[3] Drummond CJ, Fong C. Surfactant self assembly objects as novel drug delivery vehicles. current Opinion colloid Interface science. 2000;4:449-456.

[4] Sen R, Gupta R, Singh S, Mantry S, Das S. A Review on Cubosome and Virosome: the novel drug delivery system. UJPSR. 2017;3:24-33.

[5] Varde NM, Mehta NK, Thakor NM, Shah VA, Upadhyay UM. Phytosomes: a potential phospholipid nanoparticulate carrier. Pharmacie Globale (IJCP). 2012;10:1-7.

[6] \%. Drummond CJ, Fong C. Surfactant self assembly objects as novel drug delivery vehicles. current Openion colloid Interface science. 2000;4:449-456.

[7] Tilekar KB, Khade PH, Shitole MH, Jograna MB, Dr. Patil RY. Cancer Oriented Cubosomes -A Review. International Journal for Pharmaceutical Research Scholars (IJPRS). 2014;3:198210.

[8] Bhosale RR, Osmani RA, Harkare BR, Ghodake PP. Cubosomes: the Inimitable Nanoparticulate Drug Carriers. Scholars Academic Journal of Pharmacy. 2013;2:481-6.

[9] Anbarasan B, Grace XF, Shanmuganathan S. An overview of cubosomes-smart drug delivery system. Sri Ramachandra Journal of Medicine. 2015;8:1-4.

[10] Almeida JD, Brand CM, Edwards DC, Health TD: Formation of virosomes from influenza submits and liposomes. Lancet. 1975;306:899-901.

[11] Spicer PT, Lynch ML, Visscher M, Hoath S. Bicontinuous cubic liquid crystalline phase and cubosome personal care delivery systems. Personal Care Delivery Systems and Formulations. 2003.

[12] Daware SU, Saudagar RB. Formulation and Development of Cubosome Loaded Emulgel- A Review. International Journal of ChemTech Research. 2017;10:918-924.

[13] Tilekar K, Khade P, Kakade S, Kotwal S, Patil RY. "CubosomesA Drug Delivery System". International Journal of Pharmaceutical, Chemical and Biological Sciences. 2014; 4:812-824.

[14] Hundekar Y, Saboji JK, Patil SM, Nanjwade BK. Preparation and Evaluation of Diclofenac Sodium Cubosome for Percutaneous administration. World Journal of Pharmacy and Pharmaceutical Sciences. 2014;3:523-539.

[15] Jain A, Chauhan JS, Budhwani AK. Cubosome: a novel approach for nanotechnology. 2011;2:19-21.

[16] Norlén L, Al-Amoudi A. Stratum corneum keratin structure, function, and formation: the cubic rod-packing and membrane templating model. Journal of Investigative Dermatology. 2004 ;123:715-32.

[17] Esposito E, Cortesi R, Drechsler M, Paccamiccio L, Mariani P, Contado C, Stellin E, Menegatti E, Bonina F, Puglia C. Cubosome dispersions as delivery systems for percutaneous administration of indomethacin. Pharmaceutical research. 2005; 22: 2163-73.

[18] Thadanki M, Kumari PS, Prabha KS. Overview of cubosomes: a nano particle. Int J Res Pharm Chem. 2011;1:535-41.

[19] Luzzati V, Gulik Kryzwicki T, Rivas E, Reiss-Husson F, Rand RP. X-ray study of model systems: structure of the lipid-water phases in correlation with the chemical composition of the lipids. The Journal of general physiology. 1968; 51:37-43.

[20] Spicer PT, Hayden KL, Lynch ML, Ofori-Boateng A, Burns JL. Novel Process for Producing Cubic Liquid Crystalline Nanoparticles (Cubosomes). Langmuir. 2001; 17: 5748-5756.

[21] Spicer PT, Small WB, Lynch ML, Burns JL. Dry powder precursors of cubic liquid crystalline nanoparticles (Cubosomes). Journal of Nanoparticle Research. 2002; 4: 297 311.

[22] Montis C, Castroflorio B, Mendozza M, Salvatore A, Berti D, Baglioni P. Magneto cubosomes for the delivery and controlled release of therapeutics. Journal of colloid and interface science. 2015;449:317-26.

[23] Murgia S, Falchi AM, Meli V, Schillén K, Lippolis V, Monduzzi M, Rosa A, Schmidt J, Talmon Y, Bizzarri R, Caltagirone C. Cubosome formulations stabilized by a dansyl-conjugated 
block copolymer for possible nanomedicine applications. Colloids and Surfaces B: Biointerfaces. 2015;129:87-94.

[24] Lutton ES. Phase behavior of aqueous systems of monoglycerides. Journal of the American Oil Chemists Society. 1965; 42:1068-70.

[25] Kulkarni CV, Wachter W, Iglesias-Salto G, Engelskirchen S, Ahualli S. Monoolein: a magic lipid?. Physical Chemistry Chemical Physics. 2011;13: 3004-21.

[26] Boyd BJ, Whittaker DV, Khoo SM, Davey G. Lyotropic liquid crystalline phases formed from glycerate surfactants as sustained release drug delivery systems. International journal of pharmaceutics. 2006; 309: 218-26.

[27] Guillot S, Salentinig S, Chemelli A, Sagalowicz L, Leser ME, Glatter 0 . Influence of the stabilizer concentration on the internal liquid crystalline order and the size of oil-loaded monolinolein-based dispersions. Langmuir. 2010; 26: 6222-9.

[28] Chong JY, Mulet X, Boyd BJ, Drummond CJ. Steric stabilizers for cubic phase lyotropic liquid crystal nanodispersions (cubosomes). In Advances in planar lipid bilayers and liposomes 2015;21:131-187.

[29] Wadsten-Hindrichsen P, Bender J, Unga J, Engström S. Aqueous self-assembly of phytantriol in ternary systems: effect of monoolein, distearoylphosphatidylglycerol and three water-miscible solvents. Journal of colloid and interface science. 2007;315:701-13.

[30] Zhai J, Waddington L, Wooster TJ, Aguilar MI, Boyd BJ. Revisiting $\beta$-Casein as a stabilizer for lipid liquid crystalline nanostructured particles. Langmuir. 2011;27:14757-66.

[31] Uyama M, Nakano M, Yamashita J, Handa T. Useful modified cellulose polymers as new emulsifiers of cubosomes. Langmuir. 2009;25:4336-8.

[32] Spicer PT. Cubosome formation via dilution: Kinetics effects and consumer product implication. American Chemical Society. 2013; 861:346-359.

[33] Bansal S, Kashyap CP, Aggarwal G, Harikumar SL. A comparative review on vesicular drug delivery system and stability issues. Int J Res Pharm Chem. 2012;2:704-13.

[34] Rizwan SB, Dong YD, Boyd BJ, Rades T, Hook S. Characterisation of bicontinuous cubic liquid crystalline systems of phytantriol and water using cryo field emission scanning electron microscopy (cryo FESEM). Micron. 2007; 38:478-85.

[35] Bei D, Meng J, Youan BB. Engineering nanomedicines for improved melanoma therapy: progress and promises. Nanomedicine. 2010; 5:1385-99.

[36] Spicer PT. Cubosome processing industrial nanoparticle technology development. Chemical Engineering Research Design. 2005; 83:1283-86.

[37] Nanjwade BK, Hundekar YR, Kamble MS, Srichana T. Development of cuboidal nanomedicine by nanotechnology. Austin J Nanomed Nanotechnol. 2014; 2:1023.

[38] Boyd BJ. Characterisation of drug release from cubosomes using the pressure ultrafiltration method. International journal of pharmaceutics. 2003;260:239-47.

[39] Lai J, Chen J, Lu Y, Sun J, Hu F, Yin Z, Wu W. Glyceryl monooleate/poloxamer 407 cubic nanoparticles as oral drug delivery systems: I. In vitro evaluation and enhanced oral bioavailability of the poorly water-soluble drug simvastatin. AAPS PharmSciTech. 2009;10:960.

[40] Lee KW, Nguyen TH, Hanley T, Boyd BJ. Nanostructure of liquid crystalline matrix determines in vitro sustained release and in vivo oral absorption kinetics for hydrophilic model drugs. International journal of pharmaceutics. 2009;365:1909.
[41] Nguyen TH, Hanley T, Porter CJ, Boyd BJ. Nanostructured liquid crystalline particles provide long duration sustainedrelease effect for a poorly water soluble drug after oral administration. Journal of controlled release. 2011;153:180-6.

[42] Clogston J, Caffrey M. Controlling release from the lipidic cubic phase. Amino acids, peptides, proteins and nucleic acids. Journal of controlled release. 2005;107:97-111.

[43] Lynch ML, Ofori-Boateng A, Hippe A, Kochvar K, Spicer PT. Enhanced loading of water-soluble actives into bicontinuous cubic phase liquid crystals using cationic surfactants. Journal of colloid and interface science. 2003;260:404-13.

[44] Gurfinkel J, Aserin A, Garti N. Interactions of surfactants in nonionic/anionic reverse hexagonal mesophases and solubilization of $\alpha$-chymotrypsinogen A. Colloids and Surfaces A: Physicochemical and Engineering Aspects. 2011;392:322-8.

[45] Caboi F, Amico GS, Pitzalis P, Monduzzi M, Nylander T, Larsson K. Addition of hydrophilic and lipophilic compounds of biological relevance to the monoolein/water system. I. Phase behavior. Chemistry and physics of lipids. 2001;109:4762.

[46] Liu Q, Dong YD, Hanley TL, Boyd BJ. Sensitivity of nanostructure in charged cubosomes to phase changes triggered by ionic species in solution. Langmuir. 2013;29:14265-73.

[47] Shah MH, Paradkar A. Effect of HLB of additives on the properties and drug release from the glyceryl monooleate matrices. European journal of pharmaceutics and biopharmaceutics. 2007;67:166-74.

[48] Landh T. Phase behavior in the system pine needle oil monoglycerides-Poloxamer 407-water at 20. degree. The journal of physical chemistry. 1994;98:8453-67.

[49] Nakano M, Sugita A, Matsuoka H, Handa T. Small-angle X-ray scattering and 13C NMR investigation on the internal structure of "cubosomes". Langmuir. 2001 ;17:3917-22.

[50] Driever CD, Mulet X, Waddington LJ, Postma A, Thissen H, Caruso F, Drummond CJ. Layer-by-layer polymer coating on discrete particles of cubic lyotropic liquid crystalline dispersions (cubosomes). Langmuir. 2013;29:12891-900.

[51] Kwon TK, Kim JC. Complex coacervation-controlled release from monoolein cubic phase containing silk fibroin and alginate. Biomacromolecules. 2010 ;12:466-71.

[52] Deshpande S, Venugopal E, Ramagiri S, Bellare JR, Kumaraswamy G, Singh N. Enhancing cubosome functionality by coating with a single layer of poly-e-lysine. ACS applied materials \& interfaces. 2014;6:17126-33.

[53] Nilsson C, Østergaard J, Larsen SW, Larsen C, Urtti A, Yaghmur A. PEGylation of Phytantriol-Based Lyotropic Liquid Crystalline Particles. The Effect of Lipid Composition, PEG Chain Length, and Temperature on the Internal Nanostructure. Langmuir. 2014;30:6398-407.

[54] Bhosale RR, Osmani RA, Harkare BR, Ghodake PP. Cubosomes: the inimitable nanoparticulate drug carriers. Scholars Academic Journal of Pharmacy. 2013;2:481-6.

[55] Bei D, Meng J, Youan BB. Engineering nanomedicines for improved melanoma therapy: progress and promises. Nanomedicine. 2010;:1385-99.

[56] Potphode VR, Deshmukh AS, Mahajan VR. Self-Micro Emulsifying Drug Delivery System: An Approach for Enhancement of Bioavailability of Poorly Water Soluble Drugs. Asian Journal of Pharmacy and Technology. 2016;6:159-68.

[57] Hundekar YR, Saboji JK, Patil SM, Nanjwade BK. Preparation and evaluation of diclofenac sodium cubosomes for percutaneous administration. World journal of pharmacy and pharmaceutical sciences. 2014;3:523-39. 